\title{
Editorial
}

\section{The Joys of Serendipity}

By its very definition, fundamental research seeks to satisfy the researcher's curiosity without having any other aim than the desire to learn more about the world around us. Knowledge creation is the sole goal of fundamental research. By conducting fundamental research, human beings are using the scientific method to answer the question: "Why do certain phenomena occur?"

Applied research is the result of using the knowledge gained during the development of fundamental research. Applied research therefore allows us to develop and implement procedures, systems, methods, or any practices that can benefit humanity (or at least some of its subgroups).

In general, scientific discoveries are responsible for the development of fundamental research, and the progress made therein can be exploited and used in applied research. However, this sequence of events does not always occur. Sometimes, applied research pursuing a specific objective generates previously unknown knowledge.

Take, for example, the case of implantology. It can be said that the discovery of osseointegration corresponds to the definition of serendipity-an unexpected and pertinent discovery that occurs fortuitously or while one was searching for something else. This term was coined by the English bibliophile collector Horace Walpole in 1754. It is a modern version of the adage "when one does not seek, one shall find." According to Emmanuel Lemieux, in life sciences, you must cultivate a state of mind that favors making chance discoveries, but this is often repressed as scientists do not wish to give the impression that they stumbled upon their discoveries [1].

Therefore, the discovery of osseointegration sparked an exponential development in dental implantology. Some authors even cited distinct stages of the development of modern implants in the mid-1960s [2].

Clinical experimentation has shed some light on certain aspects that were initially considered outlawed or simply impossible to perform: single-step surgery, early loading, immediate implantation after dental avulsion, etc. The clinical practice of implantology has evolved and is still evolving significantly.

These advances have been primarily based on clinical research, paradigm shifts have occurred that could not have been contemplated even a few years ago. For example, immediate implant placement after dental avulsion was initially thought to inhibit bone remodeling without any clinical evidence to support this claim.

Nevertheless, and despite the unmistakable clinical achievements of modern implantology, it is unusual (to say the least) that many questions remain currently unanswered. For example, what are the reasons for marginal bone loss? We went from assuming a tolerated loss of $2 \mathrm{~mm}$ during the first year after loading an implant to fighting for a 0 -mm loss. Although this is obviously desirable, the problem is that we lack a firm grasp of the biological concepts surrounding the processes involved in this bone loss.

The more knowledge we accumulate about biological processes, the more control we will have over them. In the case of osseointegration, we have witnessed the progress of critical issues regarding implant surface condition, and some surface treatments now improve bone-implant contact (BIC) during bone healing. This has resulted in better short-term clinical results. However, our knowledge of the molecular, genetic, and cellular events occurring in the very early stages of osseointegration are very limited. Histopathologically, we only began to have reliable data when bone chambers were incorporated between the implant coils (Berglund et al. Study, 2003, for example [3]). This permitted a histopathological analysis of the first stages of healing, thereby improving our knowledge of this physiological process. It also raised new questions, such as the role that could be played by other cells, apart from osteoblasts (such as multinucleated giant cells), in obtaining and maintaining osseointegration.

A second way of formulating the question of how much control we could have over the maintenance of osseointegration is to answer the question of how to prevent this state of biological equilibrium from being lost. This can be seen with the most worrisome problem in implantology today: periimplantitis. Etiopathogenic models have inevitably been modeled on those of periodontitis, probably because of the similarities that have always been sought between a dental implants and a tooth roots. The two elements are remarkably different biologically. What unites them most is the function of supporting a genetically determined crown; this is done by both the dental roots and the artificially manufactured implants. 
Although there are many elements to design similar pathological models between the two situations, there are also multiple ways in which this pathological model does not function properly. For example, although periodontal probing is a first-order diagnostic tool in periodontitis, the value of probing as a means of assessing peri-implant health is still controversial. Thus, although biological width is a wellestablished periodontal concept, it has been documented in several studies that the biological space of implants does not respond to the same dimensions or factors as the space surrounding the tooth. Thus, peri-implantitis and periodontitis seem to be distinct pathological entities. There are even authors who claim that peri-implantitis is more like a foreignbody reaction [4].

Despite this, the pathological model of peri-implantitis modeled on that of periodontitis is still lurking in clinicians' minds.

It is not my intention in these few lines to favor one model or the other, but rather, to try to overcome this mosaic of scattered clinical data to reflect on the shortcomings on the biological processes surrounding osseointegration faced by the field of dentistry.

Fundamental research focused on the study of early vascularization of bone tissue identified a biological process that has been subsequently named as osseointegration [5]. The research contributions of many specialists have allowed unimaginable clinical development from this fundamental research, revolutionizing the oral rehabilitation of our patients in a few decades. But in spite of all this, we lack a fundamental awareness of the biological processes occurring during osseointegration maintenance, which therefore undermines the strength of scientific progress in the dentistry field.

Serendipity has undoubtedly precipitated the advent of osseointegration, and the pressure of applied science has led to significant therapeutic progress in this area. This pressure has also overshadowed the need for methodical, conscientious, and detailed biological studies of osseointegration at all levels.

I am quite convinced that, had these studies been conducted, many of the questions we are currently asking would have already been answered, with perhaps solutions to current clinical problems.
Likewise, I am convinced that current implantology must abandon any idea of exponential growth if it does not revert to focusing on the creation of sound, fundamental, biological knowledge that will help in overcoming current challenges in this area. Failure to do so would only prolong a period of limited advances, on the basis of macroscopic or histopathological features-a level of knowledge unsuited to the twenty-first century.

Of course, this knowledge cannot be sought through the prism of applied science, with a short-term vision and almost always associated with a commercial interest. Promoters of dentistry, and especially of implantology, must remember that they are health scientists. The foundations of the health sciences do not lie in the application of somewhat useful remedies, but primarily in biology and physiological processes, and then in pathological states, where the normal biology is altered.

A realistic hypothesis can only be founded on wellestablished and solid foundations. Subjected to serious scientific examination and validated by the clinical application, it can then support a stable paradigm serving as a firm foundation for the resolution of the current questions surrounding implantology.

Pr. Daniel Torres Lagares Head of Oral Surgery, University of Seville Spain, Spain

\section{References}

1. Lemieux E. The clash of ideas. Human Sciences No. 211, January 2010.

2. Buser D, Sennerby L, De Bruyn H. Modern implant dentistry based on osseointegration : 50 years of progress, current trends and open questions. Perio 2000 2017;73:7-21

3. Berglundh T, Abrahamsson I, Lang NP, Lindhe J. De novo alveolar bone formation adjacent to endosseous implants. Clin Oral Impl Res 2003; $14: 251-262$

4. Albretsson T, Camillo L, Cochran D, De Bruyn H. Periimplantitis, a complication of a foreign body or a man-made disease. Facts and fiction. Clin Impl Dent Relt Res 2016;18:840-849

5. Brånemark PI. Vital microscopy of bone marrow in rabbit. Scand J Clin Lab Invest 1959;38S:1-82 